\title{
USING TWO-PASS 3D PRE-STACK MIGRATION TO BUILD VELOCITY-DEPTH MODELS IN 3D
}

ANAT CANNING, MURALI RAMASWAMY and GERALD H.F. GARDNER HARC, Geotechnology Research Institute, 4800 Research Forest Drive, The Woodlands TX 77381, USA

\section{Introduction}

Prestack migration is a successful tool for imaging complex structures and has recently become quite popular. Experience with prestack migration indicates that the quality of the image is very sensitive to the velocity model, and therefore prestack migration is also used as a velocity analysis tool. The migrated image is usually obtained in an iterative procedure and the velocity model is updated between iterations. In other words, prestack migration and velocity estimation are related procedures. The velocity model is required so a good depth image can be obtained while the migration process itself is the tool for deriving the velocity models.

Ideally one would like to extend prestack migration to 3-D and include a 3-D velocity estimation procedure. This is a massive computation task that requires many iterations of full 3-D prestack migration and may not be very practical. A simple alternative is to use existing 2-D prestack migration tools to build 3-D velocity models. In this approach the 3-D dataset is first reorganized, using DMO techniques, as a set of parallel 2-D lines (Canning and Gardner, 1992). These lines can be processed separately and the velocity function can be obtained via $2-\mathrm{D}$ prestack depth migration applied independently to every line. However, this way 3-D effects are not taken into account and therefore the resulting velocity model is not suitable for 3-D migration.

The two-pass approach to 3-D prestack depth migration (Canning and Gardner, 1993) takes the above idea one step further. It is based on the separation of 3-D prestack Kirchhoff migration into two steps. The first step is 2-D prestack migration performed in the crossline direction; the second step is 2-D prestack migration in the inline direction. Crossline migration is done with PSI, which is a velocity independent prestack time migration (Gardner et al., 1986). Inline migration is a conventional 2-D prestack depth migration.

The two-pass approach is a useful method for building 3-D velocity models. With this approach the crossline migration is performed without any velocity information. It is therefore performed only at beginning of the process and is not repeated. In fact, this part of the process is just a preconditioning step that is used to remove 3-D effects from the data volume and position seismic energy in an approximately correct $2-\mathrm{D}$ plane. After this correction is applied depth migration, including velocity estimation, is performed in the inline direction. 


\section{Building 3-D velocity models}

The preprocessing step that was briefly described above uses DMO and PSI to reorganize the 3-D survey as a set of parallel 2-D lines free of crossline effects. At the end of this stage the data is still multi-fold and prestack depth migration can be used to build the velocity model for every line separately. This procedure does not require special 3-D migration velocity analysis and uses existing 2-D tools.

The following steps outline the procedure to build 3-D velocity models:

1. Preprocessing the 3-D data with 3-D DMO, crossline PSI and $\mathrm{DMO}^{-1}$.

2. Velocity analysis via $2-D$ prestack depth migration on selected lines through the 3-D data volume.

3. Construction of an initial 3-D velocity model by interpolation.

4. Development of a final velocity model by the application of 2-D prestack depth migration to every line in the dataset. The initial velocity model that is used in the migration comes from the interpolated velocity volume. This model is a good first guess that helps speed up the convergence process and minimizes the number of iterations required in the migration.

Our experience with the two-pass procedure indicates that when one progresses from one line to the next one, only minor changes in the model are required. Adjustment of reflector geometry is the main correction that is required between lines. The whole procedure can be carried out fairly fast.

\section{Field data example}

A 3-D dataset from the Pyrenees was used to test these ideas. The following is a comparison between two seismic depth images of the same line. One image was obtained with 2-D prestack depth migration (Figure 1) and the other with two-pass 3-D prestack migration (Figure 2). Each section was created in a separate process that included an independent model building procedure. The shallow section in this dataset is characterized by a large lateral velocity discontinuity related the shallow reflector (above 1 kilometer). Lateral velocity variations exist also between the faulted blocks at the center of the section. This survey has clear dip and strike direction and we selected the inline direction parallel to the dip direction, so structure and velocity do not vary as much in the crossline direction. Hydrocarbon targets in this survey are related to deep faulted blocks below 5 kilometers that were poorly defined on the stacked section. In Figure 2 notice the detailed image that was obtained by the migration at the region marked with an arrow. Improvement in signal to noise ratio due to focusing in 3-D can also be observed in the deeper parts of the section. A suspected buried fault, marked by a dashed line, was also brought out by the two-pass migration. The 2-D procedure was not as successful is resolving the deeper structures and generally shows poorer quality compared with the image obtained by the two-pass approach. 


\section{Discussion}

The two-pass approach to 3-D prestack migration can be used efficiently to build velocity models in 3-D as well as to obtain migrated depth images. The procedure is substantially faster than full 3-D prestack Kirchhoff migration and therefore is practical and cost effective. This procedure is not limited by size and can be used to build full velocity volumes as well as full volumes of seismic depth images.

The two-pass method is only an approximation to full 3-D prestack Kirchhoff migration. The velocity function that is obtained with this procedure is also an approximation. The most significant approximation that is embedded in the two-pass method is that the crossline migration is time migration and not depth migration. It is therefore more reliable when the subsurface structures have clear dip and strike directions. The error can be minimized by choosing the dip direction to be the inline direction. The example presented here shows a successful implementation of this procedure in a relatively complex geological setting. How useful this approach is in resolving more complex situations such as salt environments needs to be determined by experimentation. Examples of the performance of this procedure in salt environments will be presented in the workshop.

\section{Acknowledgment}

This research was carried out as part of the 3-D consortium project at the Houston Advanced Research Center (HARC). We gratefully acknowledge the support provided by the members of the HARC consortium. We would especially like to thank ELF Aquitaine (Production) which provided the 3-D dataset from the Pyrenees and gave us permission to publish the results.

\section{References}

Canning, A., and Gardner, G. H. F., 1992, Feathering correction 3-D marine data: 62nd Ann. Internat. Mtg., Soc. Expl. Geophys., Expanded Abstracts, 955-957.

Canning, A., and Gardner, G. H. F., 1993, Two-pass 3-D prestack depth migration: 63nd Ann. Internat. Mtg., Soc. Expl. Geophys., Expanded Abstracts, 892-894.

Gardner, G. H. F., Wang, S. Y., Pan, N. D., and Zhang, Z., 1986, Dip-moveout and prestack imaging: Ann. Mtg. Offshore Technology Conference, Expanded Abstracts, 75-81. 

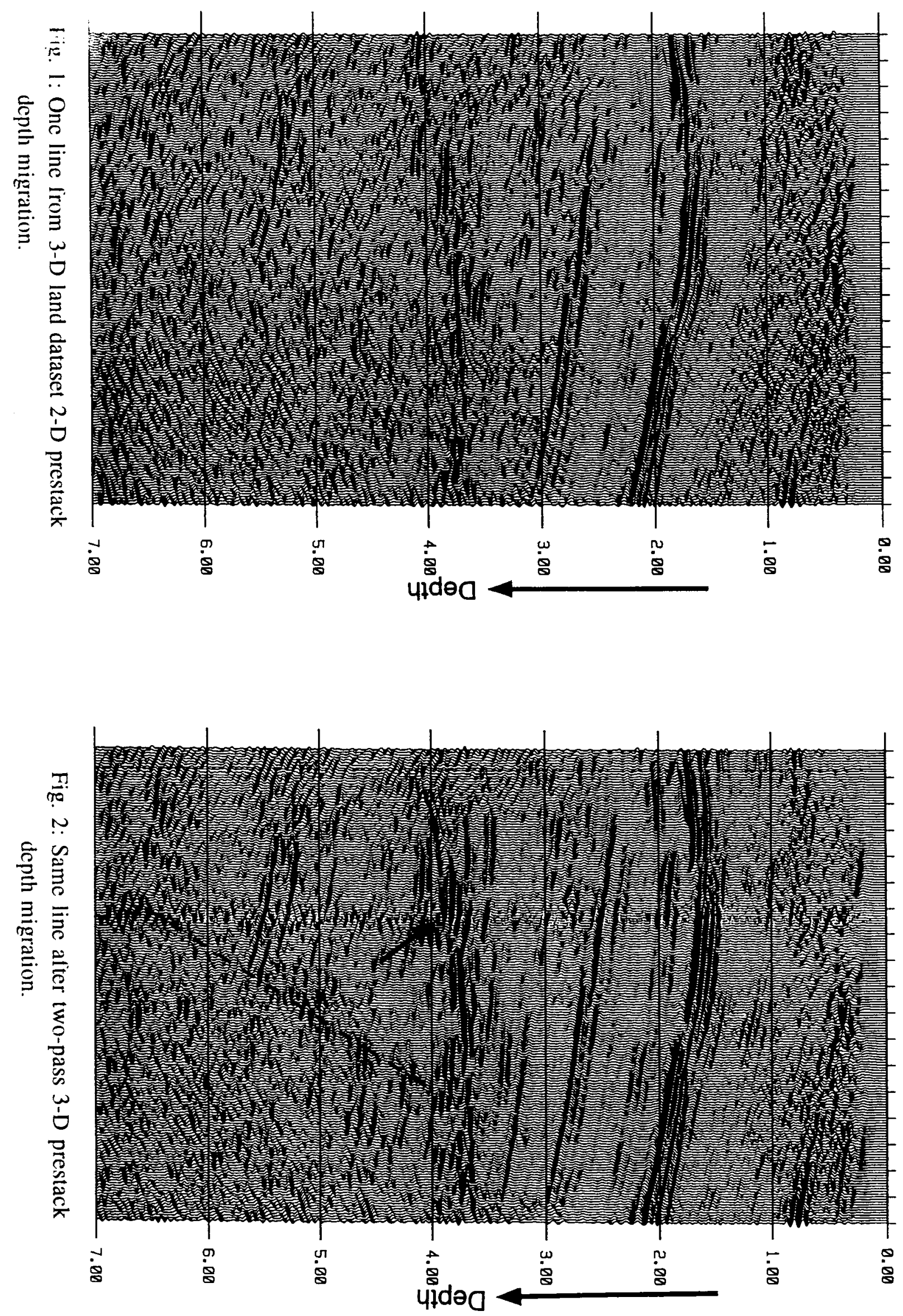